\title{
人体动脉血管的粘性流体力学模型与中心动脉 血压估计
}

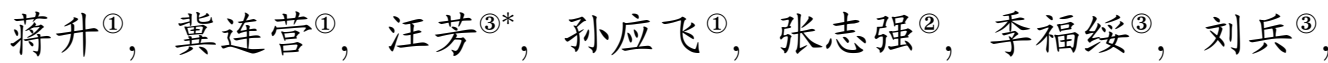
张闻多 ${ }^{3}$, 吴健康 ${ }^{(1 *}$

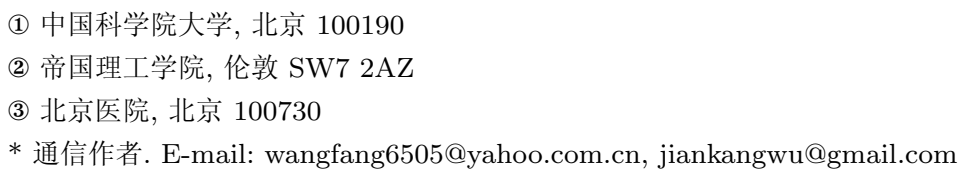

摘要 中心动脉血压与心血管疾病有着密切关联, 准确的中心动脉血压估计是心血管病, 特别是高 血压病管理的关键技术. 现有中心动脉血压估计方法, 如通用传递函数法和多点移动平均法, 均基于 临床实验和经验, 缺少系统理论基础且为通用方法, 不能排除心血管个体差异对估计结果的影响. 我 们从粘性流体力学控制方程出发, 建立了人体动脉血管网络模型, 由测量出的桡动脉起始端和末端 血压波形计算出被测者的动脉血管网络模型个性化参数, 并由此推导出升主动脉一桡动脉传递函 数, 从测出的桡动脉血压波形计算出中心动脉血压波形为区别于现有中心动脉压测算方法, 我们的 方法简称为 “模型法 (MCAP)”。该方法不仅具有理论依据, 也考虑了不同个体的心血管特性 (如: 血 管顺应性、血流阻力、血液流动惯性), 保证了估计结果的可靠性和准确性. 我们在北京医院对 50 例 被试进行中心动脉血压计算实验. 实验结果表明, 通过模型法 (MCAP) 得到的中心动脉血压波形优 于通过通用传递函数法 (GTF) 和多点移动平均法 (NPMA) 得到的结果 (与侵入式血压波形的相关 系数: $\left.r_{\mathrm{MCAP}}=0.9667, r_{\mathrm{GTF}}=0.9025, r_{\mathrm{NPMA}}=0.8099\right)$. 基于模型法计算获得的收缩压与舒张压结果 (误差均值 $(\mathrm{SBP} / \mathrm{DBP})=3.9 \mathrm{mmHg} / 3.6 \mathrm{mmHg}$, 误差标准差 $(\mathrm{SBP} / \mathrm{DBP})=7.7 \mathrm{mmHg} / 2.9 \mathrm{mmHg}$ ) 符合 AAMI 标准 (误差均值 $\leqslant 5 \mathrm{mmHg}$; 误差标准差 $\leqslant 8 \mathrm{mmHg}$ ).

关键词 血压测量 人体动脉血管模型 中心动脉血压 粘性流体力学控制方程

\section{1 引言}

中心动脉压 (Central Aortic Pressure) 指升主动脉根部血压. 2006 年, 欧洲最大规模高血压研究 ASCOT 的亚组研究 CAFE (Conduit Artery Function Evaluation) 的研究结果 [1] 显示: 中心动脉压和 中心脉压比外周肱动脉血压更能反映心脏负荷, 与心血管事件的发生更加密切相关. 近年, 中心动脉压 得到了医疗界越来越多的重视，欧洲高血压协会和欧洲心脏病协会联合发布的高血压管理指导手册已 经将中心动脉压作为血压管理的单独指标 ${ }^{[2]}$. 准确的中心动脉血压估计是心血管领域的关键技术. 
现有获取中心动脉血压的方法分为有创方式和无创方式两大类: 有创方法是指采用导管介入法直 接获取中心动脉血压. 该方法主要用于急救、心血管外科和重症监护病房等领域, 具有准确直观的优 点; 但该方法具有创伤性, 且需要专业人员进行操作, 不易大范围应用. 无创方法主要通过对桡动脉血 压波形等生理信号的分析, 估计得到中心动脉血压. 主要方法有通用传递函数 (GTF) 法 ${ }^{[3]}$ 和多点移 动平均 (NPMA: N-Point Moving Average) 法 [4]. 通用传递函数法指通过对大样本数据的分析, 估计得 到中心动脉压 一 桡动脉压的通用传递函数, 测量桡动脉血压, 利用 GTF 估计得到被试者的中心动脉 血压. 该方法已经被澳大利亚 AtCorMedical 公司的中心动脉压检测系统 (SphygmoCor) 采用. 多点移 动平均法对人体桡动脉血压波形进行 $\mathrm{N}$ 点移动平均处理 ( $\mathrm{N}$ 为采样率的四分之一) 后得到人体中心动 脉血压波形. 上述两种方法虽然得到了大量临床试验的验证, 但存在以下问题: 1) 通用传递函数法和 多点移动平均法都来自临床经验, 没有理论支撑. 2) 这两种方法均为通用方法, 不能排除个体心血管 功能的个性化差异带来的影响. 不同患者的心血管特性有着很大的不同 (如血管顺应性, 血液流动阻 力等), 这必然影响结果的准确性.

为了更准确地无创估计中心动脉血压, 解决上述现有方法的问题, 我们提出了一种基于粘性流体 力学动脉血管网络模型的人体中心动脉血压估计方法, 简称” 模型法 (MCAP: Model-based Central Aortic Pressure)”. 该方法的创新点如下: 1) 从理想弹性血管微元的粘性流体力学控制方程组出发, 完 整地提出并推导了一个可计算的、与生物医学参数一致的、具有理论基础的人体动脉血管网络模型; 2) 建立了可个性化模型参数的人体动脉血管网络模型. 通过对不同被试者桡动脉血压波形信号的分 析, 估计出对应的血管网络模型参数, 得到个性化动脉血管网络模型; 3) 首次提出了中心动脉血压波 形的理论计算方法: 使用个性化动脉血管网络模型, 计算出相应被试者的升主动脉 - 桡动脉传递函数, 由桡动脉血压波形计算出中心动脉血压波形. 实验表明, 模型法在估计中心动脉血压的准确度和稳定 性方面比现有方法具有明显的优势.

本文将在第 2 节中首先给出人体动脉血管网络模型的粘性流体力学的理论推导, 然后在第 3 节 中阐述动脉血管网络模型的参数估计、升主动脉 - 桡动脉传递函数推导, 和中心动脉血压波形计算. 和现有方法的对比实验和讨论在第 4 节中, 最后是进一步工作和展望.

\section{2 基于粘性流体力学的人体动脉血管网络模型}

本节共分 5 部分: 第 1 部分主要介绍对人体动脉血管网络模型的研究现状; 第 2 部分简要介绍了 血管建模所需要的血液动力学基础知识; 第 3 部分提出了理想弹性血管微元概念, 并从粘性流体力学 出发推导了血管微元的流体力学控制方程组; 人体大中动脉血管及小微动脉血管由上述理想弹性血管 微元级联而成, 其流体力学控制方程组的推导及建模放在第 4 部分. 本节的最后一部分介绍了由大中 动脉血管及小微动脉血管模型级联而成的人体动脉血管网络模型.

\section{1 心血管网络模型}

为了达到自桡动脉血压波形推导中心动脉血压波形的目的, 我们要建立一个可计算的动脉血管网 络模型. 首先, 它必须是可计算的, 涉及的参数不能太多, 而且必须与当前生物医学界的动脉血管参数 一致; 其次, 模型结构必须能充分地表达人体主要动脉血管的网络结构, 特别是从桡动脉到中心动脉 的血压传递函数性能; 再者, 它必须具有严谨的理论基础, 所有的假设、条件和简化都必须合理.

按照心血管系统模型的维度, 现有动脉血管的模型可分为 0-D 模型、1-D 模型、2-D 模型和 3-D 
模型 ${ }^{[5]} .3-\mathrm{D} 、 2-\mathrm{D}$ 模型考虑了血流在三维或二维空间中的流场和边界条件, 通过有限元等计算方法, 进行血流场的计算、模拟和分析 ${ }^{[6]}$. 这一类方法, 涉及参数复杂, 适用于局部血管和某些现象的分析研 究. 比如 Grinberg 等人 ${ }^{[7]}$ 建立颕内血管网络的 3-D 模型用于研究血液在不同形状和分叉血管内的血 流情况; Varghese ${ }^{[8]}$ 从计算流体力学出发, 对狭窄血管中的脉动湍流现象进行了数字模拟和分析. 3-D 模型因计算量大, 不适合本文中对全身动脉血管网络建模.

心血管系统的 1-D 模型 ${ }^{[9]}$ 考虑了血流在血管长度方向上的变化, 模型以一维流体力学控制方程 为基础. 由于计算量相对较小, 已被用于全身动脉血管建模, 如 Parker 等人 ${ }^{[10]}$ 建立了一整套心血管 系统 1-D 模型, 并用该模型对脉搏波进行时域分析; Formaggia 等人 [11] 研究了一种耦合 0-D 心脏模 型的心血管 1-D 模型. 1-D 模型的血管参数 (血管半径、血液密度、血管壁厚度等) 不能无创采集, 因 此不适合本文应用.

以 Frank ${ }^{[12]}$ 提出的 Windkessel 模型为代表的 $0-\mathrm{D}$ 模型是最早的血管模型. Westerhof ${ }^{[13]}$ 进行了 修正, 提出三参数 Windkessel 模型. Tsanas 等人 [14] 引入了级联形式的 Windkessel 模型. 0-D 模型用 等效电路来描述任意长度和任意结构的血管, 没有动脉血管网络拓扑结构; 0-D 模型基于 Windkessel 电网络模型理论, 缺少流体力学的理论基础. 因此, 也不能成为本文的选择.

为了实现我们的目标, 本文以粘性流体力学控制方程为基础, 推导理想弹性血管微元及其控制方 程, 进而推导单段人体动脉血管模型, 最后建立适用于中心动脉血压估计的人体动脉血管网络模型. 下 面, 我们将介绍: 与生物医学参数一致的动脉血管网络模型的基本参数, 基于粘性流体力学控制方程 的动脉血管模型的理论推导, 以及构建全身动脉网络拓扑结构的过程.

\section{2 血液动力学参数}

为叙述方便, 现简述血液粘性流体力学和血管的基本概念和基本参数.

1) Womersley 数. 血液的脉动特性可以用频率参数 $\alpha^{[15]}$ 来表征, 称为 Womersley 数. 频率参数 $\alpha$ 的大小与血液在血管中流动时局部惯性力与粘性力比值大小有关. 当 $\alpha$ 数较大时 $(\alpha>1)$, 血液为不定 常流, 与血液流动的粘性力相比, 由运动的非定常性引起的局部惯性力将占支配地位. 通常成人升主 动脉的 $\alpha$ 约为 $18^{[15]}$. 当 $\alpha$ 数较小时 $(\alpha<1)$, 局部惯性力的作用很小, 可以忽略不计, 此时的流动可以 近似为定常流. 通常, 成人微动脉和毛细血管的 $\alpha$ 约为 0.06 和 $0.0075^{[15]}$.

2) 血管顺应性. 血管顺应性 ${ }^{[16]}$ 又称为血管弹性, 指血管壁的缓冲能力. 血管顺应性一般用符号 $C$ 表示, 定义为容积改变量 $\mathrm{d} V$ 与相应压力改变量 $\mathrm{d} P$ 的比值, 单位为: $\mathrm{ml} / \mathrm{mmHg}$ :

$$
C=\mathrm{d} V / \mathrm{d} P \text {. }
$$

3) 血流阻力及外周阻力. 由 Poiseuille 研究的牛顿液体在管道系统内流动的规律可知血流阻力

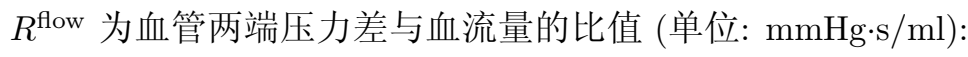

$$
R^{\text {flow }}=\frac{P\left(t, x_{\text {end }}\right)-P\left(t, x_{\text {start }}\right)}{Q(t)}=\frac{8 \eta l}{\pi(r(t))^{4}} .
$$

其中, $r$ 为血管半径, $\eta$ 为液体粘滞性, $l$ 为血管长度. 通常, 我们将小微动脉血管中的血流阻力统 称为循环系统的外周阻力. 外周阻力一般用符号 $R P$ 表示, 其公式可由公式 (2) 进一步变换为

$$
R P=\frac{P_{\mathrm{ao}, \text { mean }}-P_{\mathrm{ven}, \text { mean }}}{\mathrm{CO} / 60} \simeq \frac{P_{\mathrm{ao}, \text { mean }}}{\mathrm{CO} / 60},
$$




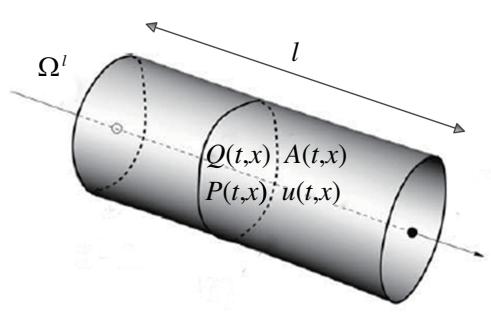

图 1 弹性血管 $\Omega^{l}$

Figure 1 Elastic vessel

其中, $P_{\mathrm{ao}, \text { mean }}$ 代表动脉血管的平均压; $P_{\mathrm{ven}, \text { mean }}$ 代表静脉血管的平均压, 可以忽略不计; $\mathrm{CO}$ 代表心 输出量, 单位是 $\mathrm{ml} / \mathrm{min}$.

4) 血液流动惯量. 血液具有一定质量, 也就具有一定的惯性, 当通过血管的血流量发生变化时, 血 液的惯性总是试图保持血液原来的运动状态, 阻碍血流的变化, 我们称这种现象为血液的惯性效应. 将 $t$ 时刻血流量变化率 $\mathrm{d} Q(t) / \mathrm{d} t$ 与血液压力差 $\Delta P(t)$ 之间的关系定义为

$$
L=\frac{\Delta P(t)}{\mathrm{d} Q(t) / \mathrm{d} t},
$$

$L$ 即为血液流动惯量, 单位为 $\mathrm{mmHg} \cdot \mathrm{s}^{2} / \mathrm{ml}$.

\section{3 理想弹性血管微元及其血液流体力学控制方程组}

定义一段弹性血管 $\Omega^{l}$, 如图 1 所示. $l$ 为血管长度; $v$ 为血管体积; $A(t, x)$ 为 $t$ 时刻, 血管 $x$ 处横 截面面积, $r(t, x)$ 为半径; $A_{0}(x)$ 为血液未流经血管时, $x$ 处的横截面面积, $r_{0}(x)$ 为半径; $u(t, x)$ 为 $t$ 时刻, 血管 $x$ 处的血流速度; $Q(t, x)$ 为 $t$ 时刻, 血管 $x$ 处的血流量. $P(t, x)$ 为 $t$ 时刻, 血管 $x$ 处的血 液压力.

如果上述弹性血管长度足够小, 即 $l=\Delta l$, 则血液粘度为定值 $\eta^{\Delta l}$, 血液密度为定值 $\rho^{\Delta l}$, 且沿着 血管长度方向血管的横截面积不变, 即 $A_{0}^{\Delta l}=A_{0}^{\Delta l}(x), A^{\Delta l}(t)=A^{\Delta l}(t, x)$, 且 $A_{0}^{\Delta l} \simeq A^{\Delta l}(t)$, 我们 称之为理想弹性血管微元, 记为 $\Omega^{\Delta l}$.

血液作为具有相当粘度的流体, 其在血管中变形、流动所遵循的规律由物理学三大守恒定律规定. 即质量守恒定律、动量守恒定律和能量守恒定律. 这三大定律对流体运动的数学描写就是流体动力学 基本方程组: 分别为连续性方程、动量方程和能量方程 ${ }^{[17]}$.

1) 由连续性方程, 可以得到下列公式 ${ }^{[6]}$ :

$$
\frac{\partial A(t)}{\partial t}+\frac{\partial Q(t, x)}{\partial x}=0 .
$$

对式 (5) 沿着血管轴线方向求导, 得到血管 $\Omega^{\Delta l}$ 所对应的连续性方程:

$$
\Delta l \frac{\mathrm{d} A^{\Delta l}(t)}{\mathrm{d} t}+Q^{\Delta l}\left(t, x_{\text {end }}\right)-Q^{\Delta l}\left(t, x_{\text {start }}\right)=0 .
$$

假设, 血管在半径方向上静态平衡, 则根据一维血管壁机械模型, 可以得到 $t$ 时刻血管 $\Omega^{\Delta l}$ 在 $x$ 处血液压力为

$$
P^{\Delta l}(t, x)=P_{\mathrm{ext}}^{\Delta l}(t, x)+\beta^{\Delta l}\left(\sqrt{A^{\Delta l}(t)}-\sqrt{A_{0}^{\Delta l}}\right),
$$


其中, $P_{\mathrm{ext}}^{\Delta l}(t, x)$ 为血管 $x$ 处, 血管壁所受到的外部压力, $\beta^{\Delta l}$ 是一个与血管壁厚度、杨氏模量及血管横 截面积有关的系数:

$$
\beta^{\Delta l}=\frac{\sqrt{\pi} h_{0}^{\Delta l} E^{\Delta l}}{\left(1-v^{2}\right) A_{0}^{\Delta l}},
$$

其中, $h_{0}^{\Delta l}$ 为血管厚度, $E^{\Delta l}$ 为血管的杨氏模量, $v$ 为值为 $1 / 2$ 的常数. 对公式 (7) 沿着血管轴线方向 积分并求时间的导数得

$$
\int_{x_{\text {start }}}^{x_{\text {end }}} \frac{\partial P^{\Delta l(t, x)}}{\partial t} \mathrm{~d} x=\int_{x_{\text {start }}}^{x_{\text {end }}} \frac{\beta^{\Delta l} \partial A^{\Delta l}(t)}{2 \sqrt{A^{\Delta l}(t)} \partial t} \mathrm{~d} x .
$$

简化上式, 因为 $A_{0}^{\Delta l} \simeq A^{\Delta l}(t)$, 可得 $t$ 时刻整段血管 $\Omega^{\Delta l}$ 受到的平均压力的变化量为

$$
\frac{\mathrm{d} \hat{P}^{\Delta l}(t)}{\mathrm{d} t}=\frac{\beta^{\Delta l}}{2 \sqrt{A_{0}^{\Delta l}}} \frac{\mathrm{d} A^{\Delta l}(t)}{\mathrm{d} t} .
$$

将公式 (10) 代入公式 (6), 得到

$$
\frac{2 \Delta l \sqrt{A_{0}^{\Delta l}}}{\beta^{\Delta l}} \frac{\mathrm{d} \hat{P}^{\Delta l}(t)}{\mathrm{d} t}+Q^{\Delta l}\left(t, x_{\text {end }}\right)-Q^{\Delta l}\left(t, x_{\text {start }}\right)=0 .
$$

2) 由动量方程, 可以得到下列公式 [18]:

$$
\frac{\partial Q(t, x)}{\partial t}+\frac{\partial}{\partial x}\left(\gamma \frac{Q(t, x)^{2}}{A(t)}\right)+\frac{A(t)}{\rho} \cdot \frac{\partial P(t, x)}{\partial x}+\frac{8 u \eta A(t)}{\rho r(t)^{2}}=0,
$$

其中, $t$ 为时间, $x$ 为血管轴线方向; $r(t)$ 为血管半径; $A(t)$ 为血管横截面积; $\eta$ 为血液粘度; $\rho$ 为血液密 度; $\gamma$ 为动量相关系数, 当均匀速度剖面时 $\gamma$ 取 $1 ; u$ 为血液流速.

在血管中, 血液的流动是不定常的脉动流, 血流参数 (速度、血管半径等) 随着血管的脉动做周期 变化. 对于理想血管 $\Omega^{\Delta l}$, 长度远小于脉动波长 (脉搏波波长), 沿着血管轴线方向, 血流量近似保持 不变, 即 $Q(t)=Q(t, x)$. 此条件下, 公式 (12) 中的第二项可以省略, 对于理想血管 $\Omega^{\Delta l}$, (12) 式沿着 血管轴线方向积分为

$$
\rho^{\Delta l} \int_{x_{\text {start }}}^{x_{\text {end }}} \frac{\partial Q^{\Delta l}(t)}{\partial t} \mathrm{~d} x+A^{\Delta l}(t) \int_{x_{\text {start }}}^{x_{\text {end }}} \mathrm{d} P^{\Delta l}(t, x)+\frac{8 u^{\Delta l} \eta^{\Delta l} A^{\Delta l}(t)}{\left(r^{\Delta l}(t)\right)^{2}} \int_{x_{\text {start }}}^{x_{\text {end }}} \mathrm{d} x=0 .
$$

又因为 $A_{0}^{\Delta l} \simeq A^{\Delta l}(t)$, 上式简化为

$$
\frac{\rho^{\Delta l} \Delta l}{A_{0}^{\Delta l}} \cdot \frac{\mathrm{d} \hat{Q}^{\Delta l}(t)}{\mathrm{d} t}+\frac{8 \eta^{\Delta l} \Delta l}{A_{0}^{\Delta l}\left(r_{0}^{\Delta l}\right)^{2}} \hat{Q} \Delta l(t) \quad+P^{\Delta l}\left(t, x_{\text {end }}\right)-P^{\Delta l}\left(t, x_{\text {start }}\right)=0 .
$$

3) 理想弹性血管微元 $\Omega^{\Delta l}$ 的流体力学控制方程组.

观察上述由流体力学连续性方程 (11) 和动量方程 (14) 系数, 由血管顺应性和血流阻力的定义 (见 式 (1), (2)), 且忽略动量方程中的粘性阻力影响, 可以得到如下结论:

$$
\begin{aligned}
& \frac{2 \Delta l \sqrt{A_{0}^{\Delta l}}}{\beta^{\Delta l}}=\frac{Q^{\Delta l}\left(t, x_{1}\right)-Q^{\Delta l}\left(t, x_{2}\right)}{\mathrm{d} \hat{P}^{\Delta l}(t) / \mathrm{d} t}=\frac{\Delta Q^{\Delta l}(t) \mathrm{d} t}{\mathrm{~d} \hat{P}^{\Delta l}(t)}=\frac{\mathrm{d} \Delta V^{\Delta l}(t)}{\mathrm{d} \hat{P}^{\Delta l}(t)}=C^{\Delta l}, \\
& \frac{8 \eta^{\Delta l} \Delta l}{A_{0}^{\Delta l}\left(r_{0}^{\Delta l}\right)^{2}}=\frac{8 \eta^{\Delta l} \Delta l}{\pi\left(r_{0}^{\Delta l}\right)^{4}}=R^{\text {flow }, \Delta l}, \\
& \frac{\rho^{\Delta l} \Delta l}{A_{0}^{\Delta l}}=\frac{P^{\Delta l}\left(t, x_{e}\right)-P^{\Delta l}\left(t, x_{s}\right)}{\mathrm{d} \hat{Q}^{\Delta l}(t) / \mathrm{d} t}=\frac{\Delta P^{\Delta l}(t)}{\mathrm{d} \hat{Q}^{\Delta l}(t) / \mathrm{d} t}=L^{\Delta l} .
\end{aligned}
$$




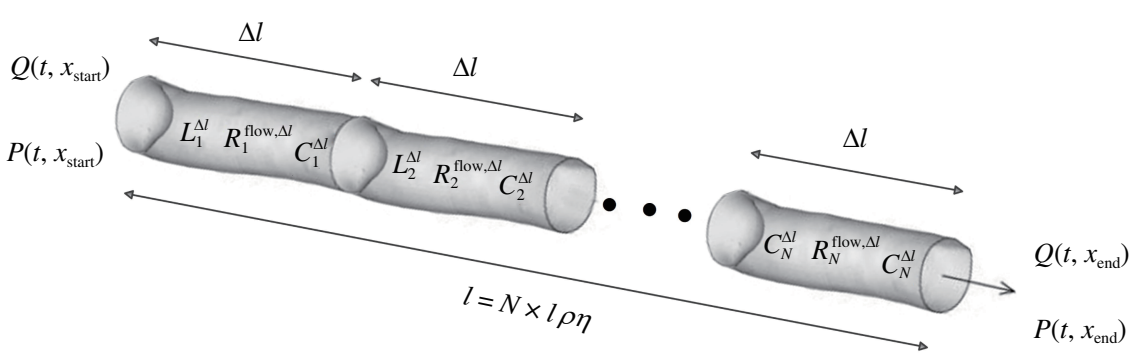

图 2 大动脉血管 $\Omega$

Figure 2 Large artery

即连续性方程 (11) 中表示血液平均压力变化率的 $\frac{\mathrm{d} \hat{P}^{\Delta l}(t)}{d t}$ 的系数 $\frac{2 \Delta l \sqrt{A_{0}^{\Delta l}}}{\beta^{\Delta l}}$ 为血管顺应性参数 $C^{\Delta l}$, 动 量方程 (14) 中表示平均流量的 $\hat{Q}^{\Delta l(t)}$ 的系数 $\frac{8 \eta^{\Delta l} \Delta l}{A_{0}^{\Delta l}\left(r_{0}^{\Delta l}\right)^{2}}$ 为血流阻力 $R^{\text {flow, } \Delta l}$. 动量方程中表示平均流 量变化率的 $\frac{\mathrm{d} \hat{Q}^{\Delta l}(t)}{\mathrm{d} t}$ 的系数 $\frac{\rho^{\Delta l} \Delta l}{A_{0}^{\Delta l}}$ 为血液流动惯量 $L^{\Delta l}$.

重新整理流体力学连续性方程和动量方程, 得到如下公式:

$$
\left\{\begin{array}{l}
C^{\Delta l} \frac{\mathrm{d} \hat{P}^{\Delta l}(t)}{\mathrm{d} t}+Q^{\Delta l}\left(t, x_{\text {end }}\right)-Q^{\Delta l}\left(t, x_{\text {start }}\right)=0, \\
L^{\Delta l} \frac{\mathrm{d} \hat{Q}^{\Delta l}(t)}{\mathrm{d} t}+R^{\text {flow }, \Delta l} \hat{Q}^{\Delta l}(t)+P^{\Delta l}\left(t, x_{\text {end }}\right)-P^{\Delta l}\left(t, x_{\text {start }}\right)=0 .
\end{array}\right.
$$

\section{4 人体动脉血管及模型}

人体动脉血管由理想弹性血管微元级联而成. 根据动脉血管横截面积的大小, 人体动脉血管分为 大中动脉血管和小微动脉血管. 我分别对这两类动脉血管及其建模进行研究.

1) 人体大中动脉血管模型.

令大中动脉血管由 $\mathrm{N}$ 段同样的理想弹性血管微元 $\Omega_{1}^{\Delta l}, \Omega_{2}^{\Delta l}, \ldots, \Omega_{N}^{\Delta l}$ 级联而成, 如图 2 所示.

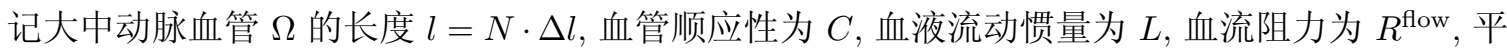
均血流量为 $\hat{Q}(t)$, 两端血流量为 $Q\left(t, x_{\text {start }}\right) 、 Q\left(t, x_{\text {end }}\right)$, 平均血压为 $\hat{P}(t)$, 两端血压为 $P\left(t, x_{\text {start }}\right)$ 、 $P\left(t, x_{\mathrm{end}}\right)$. 因为血管长度 $l$ 远小于脉搏波波长, 所以 $N$ 段等同的理想弹性血管微元的平均血压和平 均血流量近似相等. 据 (18) 式, 可推导得由 $N$ 段弹性血管微元前后级联而成的人体大中动脉血管 $\Omega$ 的流体力学控制方程组:

$$
\left\{\begin{array}{l}
C \frac{\mathrm{d} P\left(t, x_{\text {end }}\right)}{\mathrm{d} t}+Q\left(t, x_{\text {end }}\right)-Q\left(t, x_{\text {start }}\right)=0, \\
L \frac{\mathrm{d} Q\left(t, x_{\text {start }}\right)}{\mathrm{d} t}+R^{\text {flow }, l} Q\left(t, x_{\text {start }}\right)+P\left(t, x_{\text {end }}\right)-P\left(t, x_{\text {start }}\right)=0 .
\end{array}\right.
$$

上述流体力学控制方程组可用如图 3 的电路模型等效. 其中, 电路中的电压用 $P(t, x)$ 表示, 其值 等于血管中的血压值; 电路中的电流用 $Q(t, x)$ 表示, 其值等于血管中的血液流量值. 电路中的电阻用

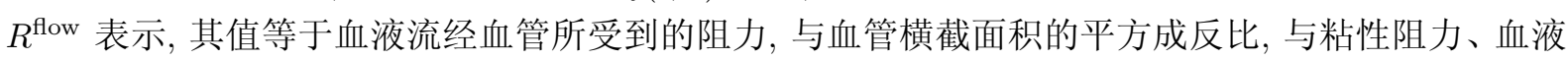
密度和血管长度成正比; 电路中的电感用 $L$ 表示, 其值等于血液流动惯量, 与血管横截面积成反比, 与 血液密度和血管长度成正比; 电路中的电容用 $C$ 表示, 其值等于血管顺应性, 与血管横截面积的开方 和血管长度成正比. 


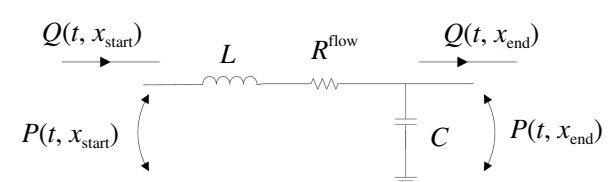

图 3 大动脉血管模型

Figure 3 Large artery model

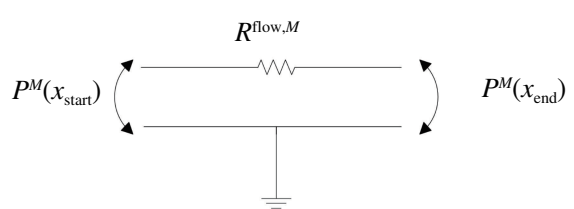

图 4 小动脉血管模型

Figure 4 Small artery model

图 3 所示模型即为一段人体大中动脉血管 $\Omega$ 的模型. 血管的长度为 $l$, 血管横截面积为 $A_{0}$, 血 液密度为 $\rho$, 血液粘度为 $\eta$, 血管顺应性为 $C=\frac{2 l \sqrt{A_{0}}}{\beta}$, 血流阻力为 $R^{\text {flow }}=\frac{8 \eta l}{\pi r_{0}^{4}}$, 血液流动惯量为 $L=\frac{\rho l}{A_{0}}$.

2) 人体小微动脉血管模型.

定义人体小微动脉血管 $\Omega^{M}$ 如下: 血管长度为 $l^{M}$, 且血管长度远小于血液流动形成的脉搏波波 长; 血管横截面积沿血管长度方向不变, 即 $A_{0}^{M}=A_{0}^{M}(x), A^{M}(t)=A^{M}(t, x)$, 且 $A_{0}^{M} \simeq A^{M}(t)$; 血液 粘度为定值 $\eta^{M}$; 血液密度为定值 $\rho^{M}$; 血管顺应性为 $C^{M}$; 血液流动惯量为 $L^{M}$; 血流阻力为 $R^{\text {flow }, M}$; 随着时间 $t$ 的变化, 血管中血液的流量和压力保持不变, 即血液在血管中的流动为定常流动; 平均血 流量为 $\hat{Q}(t)$; 血管两端血流量为 $Q^{M}\left(x_{\mathrm{start}}\right), Q^{M}\left(x_{\mathrm{end}}\right)$; 血管平均血压为 $\hat{P}^{M}$; 两端血压为 $P^{M}\left(x_{\mathrm{start}}\right)$, $P^{M}\left(x_{\text {end }}\right)$.

同样, 人体小微动脉血管 $\Omega^{M}$ 可由 $N$ 段理想弹性血管微元 $\Omega_{1}^{M, \Delta l}, \Omega_{2}^{M, \Delta l}, \ldots, \Omega_{N}^{M, \Delta l}$ 级联而成. 进而得到小微动脉血管 $\Omega^{M}$ 的流体力学控制方程:

$$
\left\{\begin{array}{l}
C^{M} \frac{\mathrm{d} P^{M}\left(x_{\text {end }}\right)}{\mathrm{d} t}+Q^{M}\left(x_{\text {end }}\right)-Q^{M}\left(x_{\text {start }}\right)=0, \\
L^{M} \frac{\mathrm{d} Q^{M}\left(x_{\text {start }}\right)}{\mathrm{d} t}+R^{\text {flow }, M} Q\left(x_{\text {start }}\right)+P^{M}\left(x_{\text {end }}\right)-P^{M}\left(x_{\text {start }}\right)=0 .
\end{array}\right.
$$

因为血液在小微动脉血管中是定常流, 所以上述方程组可进一步简化得

$$
\left\{\begin{array}{l}
Q^{M}\left(x_{\text {end }}\right)-Q^{M}\left(x_{\text {start }}\right)=0, \\
R^{\text {flow }, M} Q\left(x_{\text {start }}\right)+P^{M}(x)-P^{M}\left(x_{\text {start }}\right)=0 .
\end{array}\right.
$$

式 (21) 可用电路模型等效, 得到人体小微动脉血管模型, 见图 4. 其中, 电路中的电压用 $P^{M}(x)$ 表示, 其值等于血管系统中的血压值; 电路中的电流用 $Q^{M}(x)$ 表示, 其值等于血管系统中的血液流量 值. 电路中的电阻用 $R^{\mathrm{flow}, M}$ 表示, 其值 $R^{\mathrm{flow}, M}=\frac{8 \eta^{M} l^{M}}{\pi\left(r_{0}^{M}\right)^{4}}$.

\section{5 人体动脉血管网络模型}

人体动脉血管网遍布全身, 沿着血流方向, 动脉血管从升主动脉出发, 通过分叉不断延生到身体 各部位的小微动脉. 参考 Stergiopulos ${ }^{[19]}$ 和 Wang ${ }^{[20]}$, 我们以分叉结构组成包含大中动脉血管网络和 小微动脉血管网络的全身的动脉血管网络.

根据大中动脉血管网络的分叉结构和血管几何参数 (血管半径 $r$ 、血管长度 $l$ 、血管壁厚度 $h$ 及杨氏模量 $E$ ), 可以将大中动脉血管网络分为 55 段大中动脉血管, 文献 [20,21] 给出了它们的生理 参数表. 每段大中动脉血管沿着血管轴线方向横截面积近似不变. 人体大中动脉血管脉搏波波速约为 $8 \mathrm{~m} / \mathrm{s}$, 以 1 秒为脉搏波周期计算, 人体脉搏波波长约为 $8 \mathrm{~m}$, 远大于人体大中动脉血管长度: 人体最 


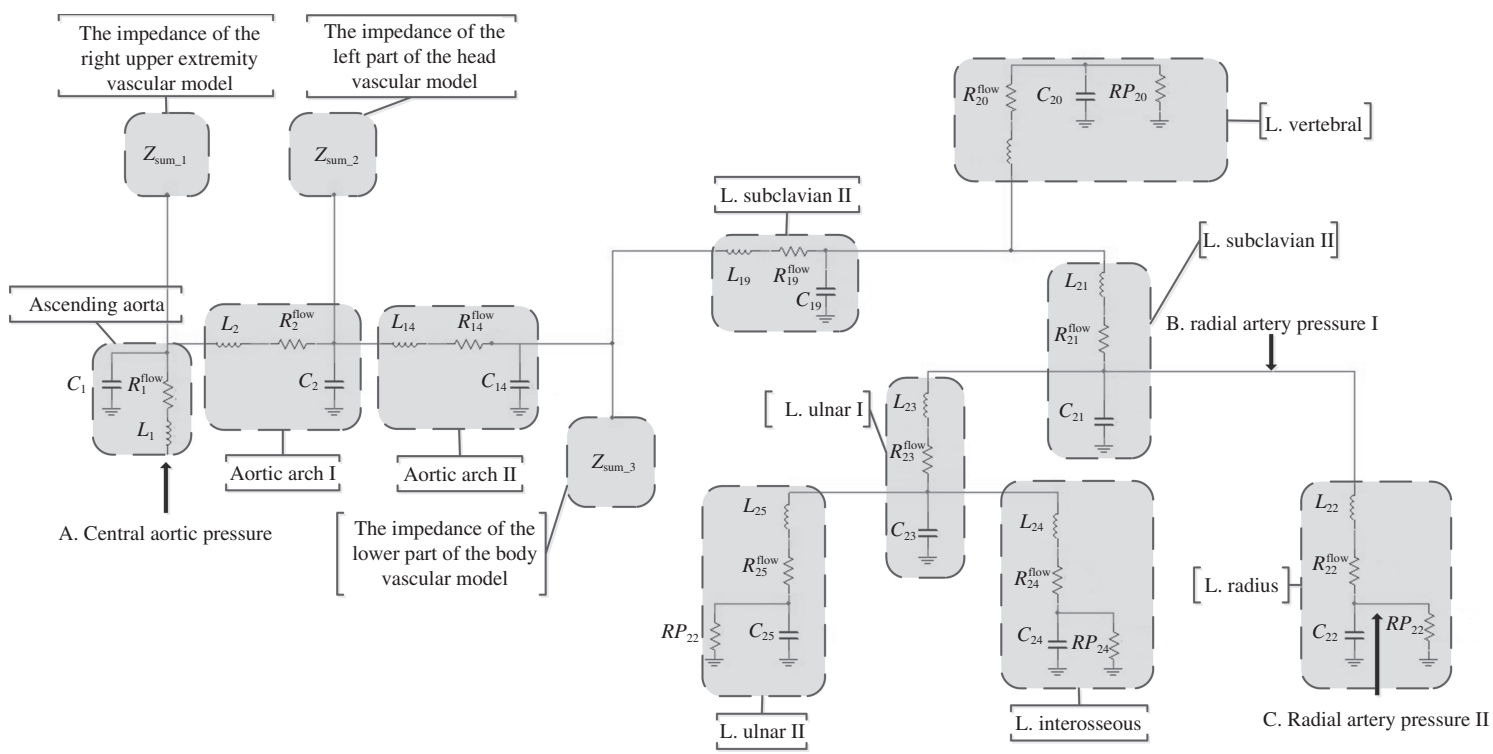

图 5 升主动脉 - 桡动脉血管网络模型

Figure 5 The model of ascending aorta-radial artery

长动脉血管为股动脉, 长度约为 $0.44 \mathrm{~m}$. 上述 55 根大中动脉血管组成的大中动脉血管网络模型可以 由如图 3 所示的大中动脉血管模型 $\Omega_{1}, \Omega_{2} \ldots, \Omega_{55}$ 的网络结构表示. 篇幅所限, 我们将升主动脉 - 桡 动脉血管网络模型绘制于图 5. 为了简化表示, 图中, 我们将由图 4 小微动脉血管模型组成的每一个 小微动脉血管网络模型用一个外周阻力 $R P$ 表示.

\section{3 基于动脉血管网络模型的中心动脉血压估计}

本节分为 3 部分: 第 1 部分为动脉血管网络模型的模型参数估计方法, 该方法主要基于桡动脉脉 搏波波形分析; 基于上述血管网络模型的中心动脉 - 桡动脉血压传递函数推导放在本节的第 2 部分; 第 3 部分推导了基于传递函数的中心动脉血压估计方程.

\section{1 动脉血管网络模型的参数估计}

对于正常人, 全身外周阻力与各动脉血管模型所级联的外周阻力的比值 $\omega_{i}^{R P}$ 为常数, 可由血管 参数 $[20,21]$ 计算得到. 现在, 我们得到人体动脉血管网络模型中 $j$ 号血管模型所级联的外周阻力 $R P_{j}(j=6,8,10, \ldots, 55)$ :

$$
R P_{j}=\frac{R P}{1-\sum_{i} \frac{R P}{R P_{i}}}=\frac{R P}{1-\sum_{i} w_{i}^{R P}}
$$

其中, $i=6,8, \ldots, 55(i \neq j)$.

同一个体, 不同动脉血管中血液密度 $\rho$ 和血液粘度 $\eta$ 近似不变; 对于正常人, 血管 $\Omega_{i}, \Omega_{j}$ 的血管 长度、血管横截面积 (血管半径)、血管厚度及杨式模量 $\left(l, A, r, h_{0}, E\right)$ 的比值可以近似为定值, 记为 $\omega_{i j}^{A}, \omega_{i j}^{l}, \omega_{i j}^{r}, \omega_{i j}^{h_{0}}, \omega_{i j}^{E}$, 其值可以由血参数 ${ }^{[22,23]}$ 计算得到.

由 2.3 小节可知, 对于图 5 中的每一根大中动脉血管模型的血管顺应性为 $C_{i}=\frac{2 l_{i} \sqrt{A_{0, i}}}{\beta_{i}}$ (其中 $\left.\beta_{i}=\frac{\sqrt{\pi} h_{0, i} E_{i}}{\left(1-v^{2}\right) A_{0, i}}\right)$, 血流阻力为 $R_{i}^{\text {flow }}=\frac{8 \eta l_{i}}{\pi r_{i}^{4}}$, 血液流动惯量为 $L_{i}=\frac{\rho l_{i}}{A_{0, i}}$, 其中 $i=1,2,3, \ldots, 55$. 因此, 如 


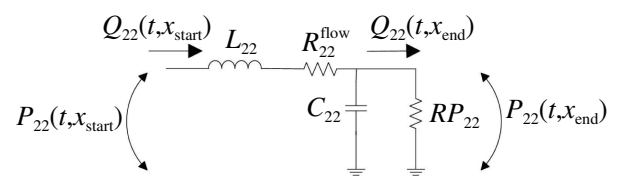

图 6 桡动脉血管网络模型

Figure 6 The model of radial artery

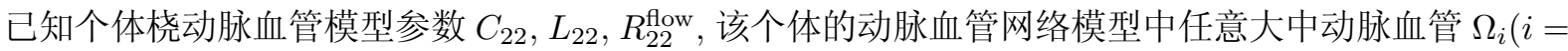
$1,2, \ldots, 55)$ 的模型参数 $L_{i}, C_{i}, R_{i}^{\text {flow }}$ 都可以用下列公式估计:

$$
\left\{\begin{array}{l}
C_{i}=C_{22} \cdot \frac{l_{i} \sqrt{A_{i}} \beta_{22}}{l_{22} \sqrt{A_{22}} \beta_{i}}=\frac{C_{22} \omega_{i, 22}^{l}\left(\sqrt{\omega_{i, 22}^{A}}\right)^{3}}{\omega_{i, 22}^{h_{0}} \omega_{i, 22}^{E_{0}}}, \\
R_{i}^{\text {flow }}=R_{22}^{\text {flow }} \frac{l_{i} /\left(r_{i}\right)^{4}}{l_{22} /\left(r_{22}\right)^{4}}=R_{22}^{\text {flow }} \omega_{i, 22}^{l} /\left(\omega_{i, 22}^{r}\right)^{4}, \\
L_{i}=L_{22} \frac{l_{i} / A_{0, i}}{l_{22} / A_{0,22}}=L_{22} \omega_{i, 22}^{l} / \omega_{i, 22}^{A} .
\end{array}\right.
$$

将图 5 中的左桡动脉血管模型单独绘制如图 6. 下面我们讨论桡动脉血管模型参数的测量和估计 方法.

1) 左桡动脉血管模型 $R P_{22}$ 估计.

根据式 (22) 可以得到左桡动脉血管模型所级联的外周阻力 $R P_{22}$ :

$$
R P_{22}=\frac{R P}{1-\sum_{i} w_{i}^{R P}} .
$$

2) 左桡动脉血管模型参数 $L_{22}, C_{22}, R_{22}^{\text {flow }}$ 估计.

根据图 6 所示桡动脉血管模型, 我们可以得到如下数学表达式:

$$
\left\{\begin{array}{l}
P_{22}\left(t, x_{\text {end }}\right)=Q_{22}\left(t, x_{\text {end }}\right) R P_{22}, \\
\frac{\mathrm{d} P_{22}\left(t, x_{\text {end }}\right)}{\mathrm{d} t}=\frac{Q_{22}\left(t, x_{\text {start }}\right)-Q_{22}\left(t, x_{\text {end }}\right)}{C_{22}}, \\
\frac{\mathrm{d} Q_{22}\left(t, x_{\text {start }}\right)}{\mathrm{d} t}=\frac{P_{22}\left(t, x_{\text {start }}\right)-R_{22}^{\text {flow }} Q_{22}\left(t, x_{\text {start }}\right)-Q_{22}\left(t, x_{\text {end }}\right)}{L_{22}} .
\end{array}\right.
$$

整理式 $(25)$ 得

$$
\begin{aligned}
& L_{22} C_{22} R P_{22}\left(\frac{d Q_{22}\left(t, x_{\text {end }}\right)}{d t}\right)^{2}+\left(L_{22}+R_{22}^{\text {flow }} C_{22} P R_{22}\right) \cdot \frac{d Q_{22}\left(t, x_{\text {end }}\right)}{d t} \\
& +\left(R P_{22}+R_{22}^{\text {flow }}\right) Q_{22}\left(t, x_{\text {end }}\right)=P_{22}\left(t, x_{\text {start }}\right) .
\end{aligned}
$$

在已知 $Q_{22}\left(t, x_{\mathrm{end}}\right), P_{22}\left(t, x_{\mathrm{start}}\right)$ 的前提下, 我们通过最小二乘估计算法估计得到公式 $(26)$ 的参 数矩阵 $\hat{\theta}=\left[a_{1}, a_{2}, a_{3}\right]$, 进而得到参数 $L_{22}, C_{22}, R_{22}^{\text {flow }}$ 的表达式:

$$
\left\{\begin{array}{l}
R_{22}^{\text {flow }}=a_{3}-R P_{22}, \\
C_{22}=\frac{a_{2} R P_{22}+\sqrt{\left(a_{2} R P_{22}\right)^{2}-4 a_{1} R_{22}^{\text {flow }}\left(R P_{22}\right)^{2}}}{2 R_{22}^{\text {flow }}\left(R P_{22}\right)^{2}}, \\
L_{22}=a_{2}-C_{22} R_{22}^{\text {flow }} R P_{22} .
\end{array}\right.
$$




\section{2 基于动脉血管网络模型的传递函数}

图 5 所示人体动脉血管网络模型中的任意血管的血压传递函数记为: $T F_{i}(s)$. 观察模型, 我们可以 得到如下传递函数:

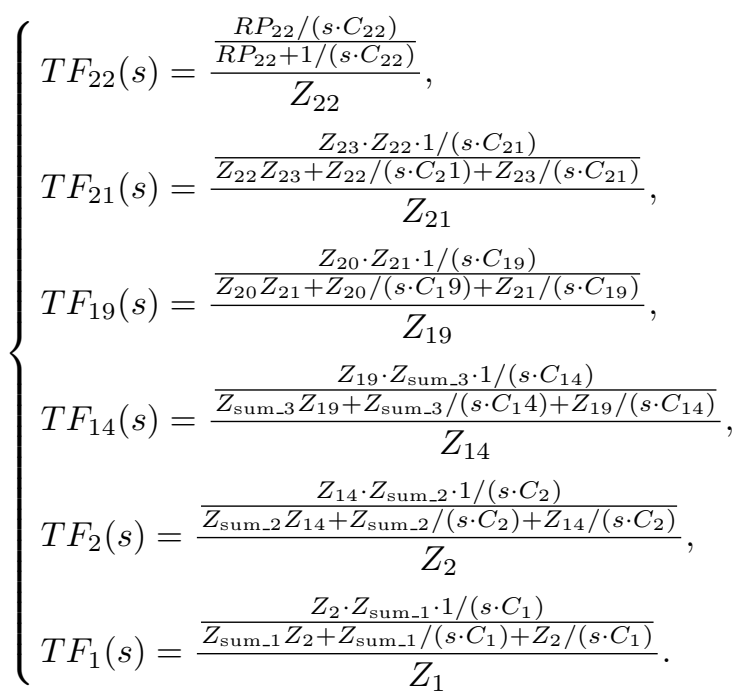

式 (28) 的 $Z_{\text {sum_1 }}$ 为图 5 所示动脉血管网络模型中右上肢动脉血管网络模型的特征阻抗. $Z_{\text {sum_2 }}$ 为图 5 所示动脉血管网络模型中做左侧头部动脉血管网络模型的特征阻抗. $Z_{\mathrm{sum} \_3}$ 为图 5 所示动脉 血管网络模型中下半身动脉血管网络模型的特征阻抗. $Z_{i}$ 为相应血管的特征阻抗. 上述特征阻抗的 推导因篇幅所限, 略为不表.

整理式 (28) 中的传递函数, 得到以升主动脉的始端的血压 (图 5 中的 A 点) 为输入, 以桡动脉模 型的末端的血压 (图 5 中的 $\mathrm{C}$ 点) 为输出的传递函数 $T F(s)$.

$$
T F(s)=T F_{22}(s) \cdot T F_{21}(s) \cdot T F_{19}(s) \cdot T F_{14}(s) \cdot T F_{2}(s) \cdot T F_{1}(s)
$$

\section{3 中心动脉血压估计}

在血液循环过程中, 当心室收缩主动脉瓣打开, 血液射入主动脉内, 由于血管系统有阻力, 使射入 主动脉的血液暂时留在主动脉近端, 并引起主动脉扩张、压力升高. 当心室舒张、主动脉瓣关闭、射血 停止, 主动脉将因弹性恢复而收缩. 主动脉这种一张一缩, 压力将从升高区域开始以波的形式向主动 脉远端及其分支传播, 连续地使远端的动脉扩张, 亦即从主动脉压出的血液将以压力波动的形式在动 脉系统内传播. 这种压力波动的形式称为脉搏波.

桡动脉血压波形和中心动脉血压波形 $c B P$ 之间的关系可以在频率域用传递函数 $T F(\omega)$ 表示. 中 心动脉血压估计公式如下:

$$
c B P(\omega)=r B P(\omega) / T F(\omega)
$$

\section{4 实验及分析}

\section{1 中心动脉血压估计实验}

为了验证采用 “模型法” 估计中心动脉血压的有效性, 我们与北京医院心内科合作, 以侵入式方法 
表 1 侵入式血压采集实验的被试信息

Table 1 The subjects' information

\begin{tabular}{ccc}
\hline Property & Mean \pm SD (or \%) & Range \\
\hline Age & $65.3 \pm 9.3$ & $49 \sim 83$ \\
Gender & Man $45.5 \%$, Woman $54.5 \%$ & \\
SBP (mmHg) & $130.2 \pm 21.1$ & $82 \sim 176$ \\
DBP $(\mathrm{mmHg})$ & $66.6 \pm 13.9$ & $44 \sim 93$ \\
\hline
\end{tabular}

依次采集中心动脉血压波形、桡动脉起始端血压波形和桡动脉末端血压波形, 以桡动脉血压波形作为 模型法的输入信号, 以中心动脉血压波形作为评估模型法中心动脉压估计方法的金标准. 同时, 我们比 较了模型法 (MCAP) 与通用传递函数法 (GTF) 及多点移动平均法 (NPMA). 我们对 50 例被试进行 实验 (被试情况见表 1). 每例共采集中心动脉压波形、桡动脉起始端和末端波形各 10 个. 3 个采集点 顺序采集, 时间差在 2 分钟左右. 我们假设在脉搏波采集过程中, 血压变化很小. 为了减小脉搏间差异 所引起的实验误差，我们在 10 个脉搏波波形中选取最接近平均值的波形进行实验：即计算它们的相 似度量, 选取与所有其它波形相似度最小的波形.

具体比较方法为: (a) 建立被试的动脉血管网络模型, 由分析桡动脉起始端和末端血压, 获得动脉 血管网络模型参数; (b) 计算升主动脉 - 桡动脉的传递函数; (c) 采用式 (30) 计算中心动脉压结果, (d) 用 NPMA 法 (N=Hz/4) 和 GTF 法 (频率响应参考文献 ${ }^{[22]}$ ) 计算中心动脉压. (g) 绘制桡动脉末端血 压波形; 传递函数幅频响应; 中心动脉血压波形. (h) 比较通过 MCAP、GTF 和 NPMA 法计算的中心 动脉血压波形与侵入式血压波形的相似程度 (用相关系数表示). (i) 对上述方法得到的中心动脉收缩 压与舒张压进行统计学分析. 图 7 中所示为从 50 例中随机选取的 2 例实验结果.

中心动脉脉搏波波形含有丰富的心血管信息, 比如中心动脉脉搏波的上升支反应了心肌收缩能力; 下降支的切记点反应主动脉瓣关闭的时间; 由脉搏波波形计算得到的中心动脉反射波增强指数 (AIX) 与脉搏波波速和动脉硬化水平直接相关等. 我们认为中心动脉波形的还原程度是检验中心动脉估计 方法的一个重要指标. 我们以侵入式血压测量是获得的中心动脉血压作为的金标准. 我们随机采集 50 例被试结果中侵入式升主动脉血压波形与基于 MCAP 法模型法、GTF 法及 NPMA 法多点移动平均 法计算得到的中心动脉血压波形的 150 个采样点进行相关性分析, 进而评估模型法 MCAP 法、GTF 法及 NPMA 法在估计中心动脉血压波形方面的有效性 (见图 8).

同时, 中心动脉的收缩压与舒张压是衡量高血压水平的重要指标. 为了比较 MCAP 与 GTF、NPMA 及侵入式测量的中心动脉收缩压与舒张压值, 我们对上述方法得到的中心动脉收缩压、舒张压值进行 统计学分析 (见表 2).

观察图 7 的 (c) 和 (f) 可知: 模型法计算结果包含了丰富的脉搏波下降支信息, 比如: 被试的模型 法计算结果均没有丢失脉搏波下降支切记点.

同时, 基于模型法计算得到的中心动脉血压波形的上升支陡峭程度与侵入式血压波形的上升支陡 峭程度最接近.

上述结论在对图 7 结果的相关性分析中得到了印证：基于模型法计算的中心动脉血压波形与侵 入式升主动脉血压波形之间相似度最高, GTF 法的结果相识程度次之, 多点移动平均法结果的相关识 程度最差 (与侵入式血压波形的相关系数 $\left.r_{\mathrm{MCAP}}=0.9667, r_{\mathrm{GTF}}=0.9025, r_{\mathrm{NPMA}}=0.8099\right)$.

中心动脉收缩压与舒张压是评估血压水平的重要指标. 我们比较基于 4 种方法获得的中心动脉收 


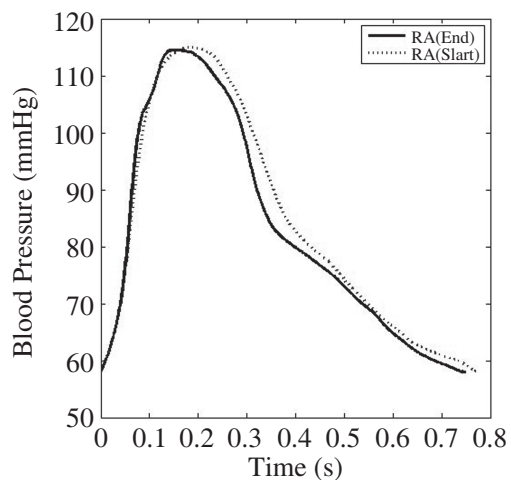

(a)

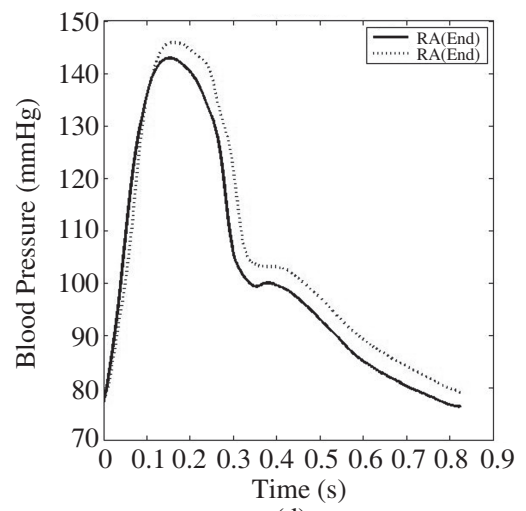

(d)

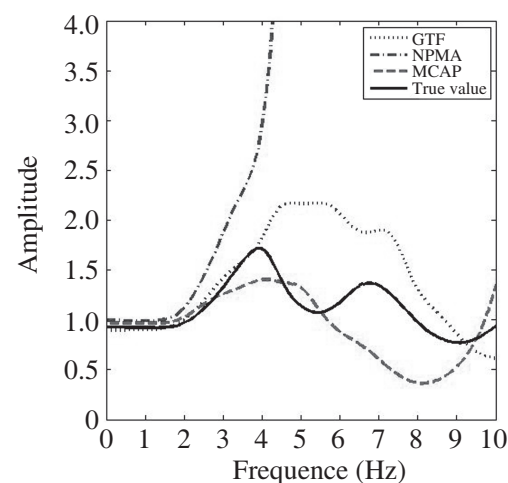

(b)

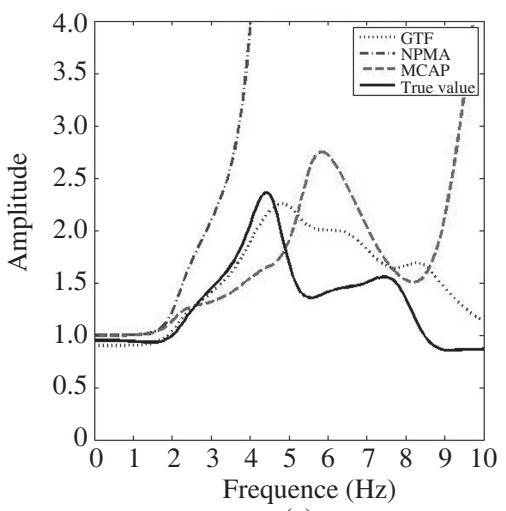

(e)

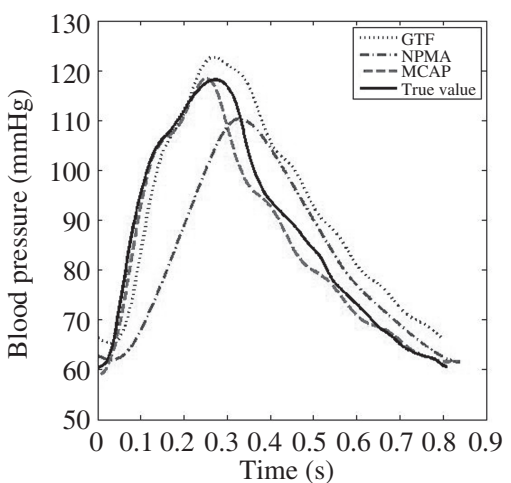

(c)

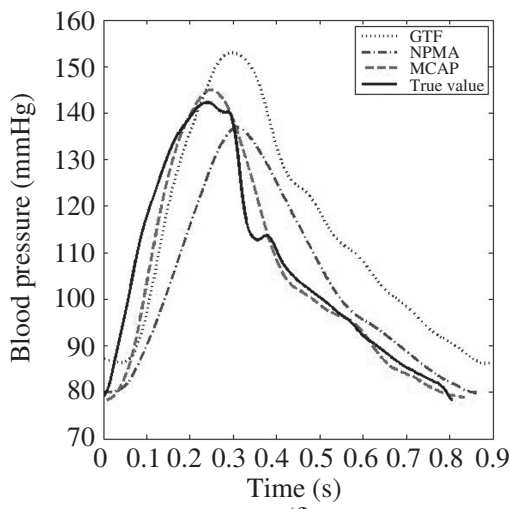

(f)

图 7 图 (a) (c) 为 ID 为 421853 的被试的中心动脉血压计算结果; 图 (d) (f) 为 ID 为 421931 的被试的中 心动脉血压计算结果. (a), (d) 为桡动脉末端血压; (b), (e) 为 GTF, NPMA, MCAP 与侵入式血压测量的中心 动脉 一 桡动脉传递函数幅频响应; (c), (f) 为 GTF 法, NPMA, MCAP 计算的中心动脉压与侵入式血压测量 的升主动脉根部血压比较

Figure 7 (a) Blood pressure waveform of radial artery; (b) amplitude-frequency response of transfer function; (c) blood pressure waveform of central artery; (d) blood pressure waveform of radial artery; (e) amplitude-frequency response of transfer function; (f) blood pressure waveform of central artery. (a)-(c) is the estimated results about the subject's (ID is 421853) central arterial blood pressure, (d)-(f) is the estimated results about the subject's (ID is 421931) central arterial blood pressure. (a), (d) are the blood pressure waveforms of radial artery; (b), (d) are the amplitude-frequency responses of transfer function; (c), (f) are the blood pressure waveforms of central artery

缩压与舒张压: 模型法的收缩压计算结果最准确 (误差均值 $(\mathrm{MCAP})=3.9 \mathrm{mmHg}$, 误差均值 $(\mathrm{GTF})=6.0$ $\mathrm{mmHg}$, 误差均值 $(\mathrm{NPMA})=7.7 \mathrm{mmHg}$ ), 稳定性最高 (误差标准差 $(\mathrm{MCAP})=7.7 \mathrm{mmHg}$, 误差标准差 $(\mathrm{GTF})=8.1 \mathrm{mmHg}$, 误差标准差 $(\mathrm{NPMA})=8.6 \mathrm{mmHg})$; 模型法的舒张压计算结果稳定性最高 (误差均 值 $(\mathrm{MCAP})=2.9 \mathrm{mmHg}$, 误差均值 $(\mathrm{GTF})=3.4 \mathrm{mmHg}$, 误差均值 $(\mathrm{NPMA})=4.0 \mathrm{mmHg}) ; \mathrm{NPMA}$ 法的舒张压计算结果最准确 (误差均值 $(\mathrm{MCAP})=3.6 \mathrm{mmHg}$, 误差均值 $(\mathrm{GTF})=5.7 \mathrm{mmHg}$, 误差均值 $(\mathrm{NPMA})=2.1 \mathrm{mmHg})$. 综合来看, 基于模型法的收缩压与舒张压计算结果最优, 且该方法符合 AAMI 标准 (误差均值 $\leqslant 5 \mathrm{mmHg}$; 误差标准差 $\leqslant 8 \mathrm{mmHg}$ ).

由公式 (30) 可知, 中心动脉血压波形受中心动脉 — 桡动脉传递函数影响. 由 $\mathrm{Chen}^{[22]}$ 的研究可 知, 脉搏波的功率谱密度主要集中分布在 $0 \sim 5 \mathrm{~Hz}$ 范围内, 又因为图 7 中脉搏波周期约为 $0.6 \sim 0.7 \mathrm{~s}$ (频 率为 $1.7 \sim 1.4 \mathrm{~Hz})$, 因此可以近似认为中心动脉血压的收缩压、舒张压和脉压差主要由传递函数 $0 \sim 2 \mathrm{~Hz}$ 

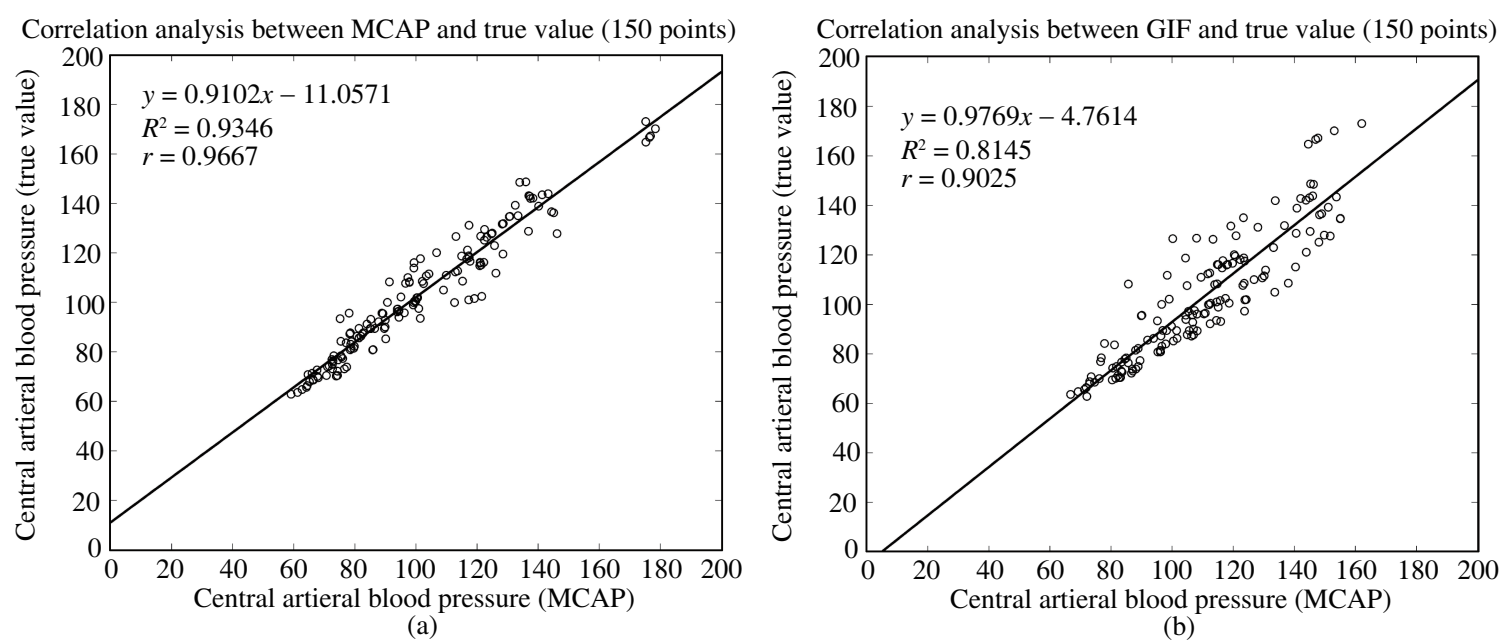

(a)

Correlation analysis between NPMA and true value (150 points)

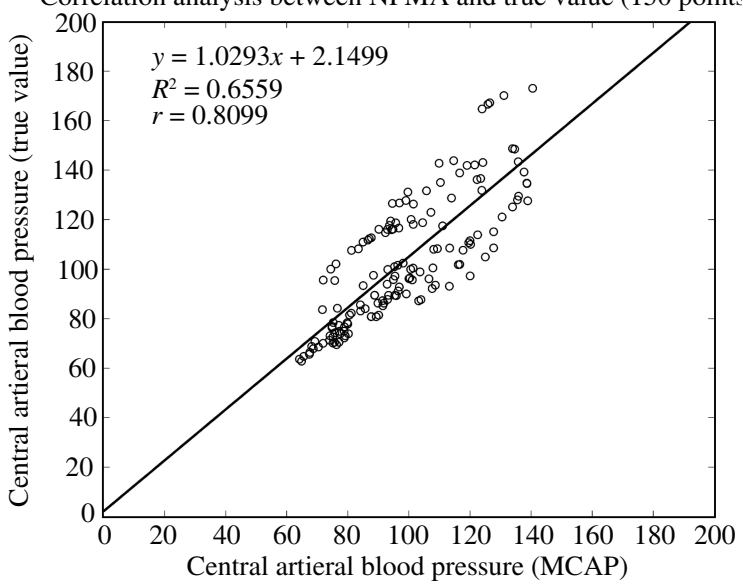

(c)

图 8 中心动脉血压的真实值与估计值之间的相关性分析. (a) 是中心动脉血压的真实值与通过 MCAP 法得到的 估计值之间的相关性分析)；(b) 是中心动脉血压的真实值与通过 GTF 法得到的估计值之间的相关性分析)；(c) 是 中心动脉血压的真实值与通过 NPMA 法得到的估计值之间的相关性分析

Figure 8 Correlation analysis about central arterial blood pressure. (a) The correlation analysis between MCAP and true value; (b) the correlation analysis between GTF and true value; (c) the correlation analysis between NPMA and true value

范围内幅频响应决定. 传递函数 $2 \sim 5 \mathrm{~Hz}$ 范围内的幅频响应则决定了脉搏波波形变化 (如切记点位置, 反射波位置, 上升支斜率等). 表 3 为模型法、GTF 法及多点移动平均方法所对应的中心动脉 一 桡动 脉传递函数的幅频响应与侵入式方法对应的中心动脉 — 桡动脉传递函数幅频响应的统计学分析. 由 分析可知, 模型法所对应的传递函数幅频响应要优于 GTF 法和多点移动平均法的传递函数幅频响应.

\section{2 个体差异分析}

血压受血管特征参数, 如: 血管顺应性、血流阻力和血液流动惯量影响. 不同被试因年龄、性别 和疾病的不同有着不同的心血管特征参数. MCAP 法区别于 GTF 法和 NPMA 法的一大特点在于 MCAP 法在估计中心动脉血压时考虑了不同患者血管顺应性、血流阻力和血液流动惯量的影响. 我们 以 ID 为 10202 和 317878 的被试为例分析个体差异对心血管网络模型参数的影响 (图 9). 
表 2 舒张压和收缩压的统计学分析

Table 2 Statistical analysis about DBP and SBP ((a) is statistical analysis about DBP and (b) is statistical analysis about SBP)

(a) DBP $(\mathrm{mmHg})$

\begin{tabular}{lccccc}
\hline & Mean & SD & Mean difference & SD difference & CI (95\%) \\
\hline Real & 70.4 & 9.6 & & & 2.9 \\
MCAP & 66.8 & 9.2 & 3.6 & 3.4 & 2.5 \\
GTF & 76.1 & 9.4 & 5.7 & 4.0 & 2.8 \\
NPMA & 72.4 & 9.4 & 2.1 & 2.0 \\
\hline
\end{tabular}

(b) SBP $(\mathrm{mmHg})$

\begin{tabular}{lllccc}
\hline & Mean & SD & Mean difference & SD difference & CI $(95 \%)$ \\
\hline Real & 135.6 & 19.2 & & & 7.7 \\
MCAP & 139.5 & 17.6 & 3.9 & 8.1 & 5.5 \\
GTF & 141.6 & 15.4 & 6.0 & 8.6 & 5.8 \\
NPMA & 127.9 & 13.6 & 7.7 & 6.1 \\
\hline
\end{tabular}

表 3 传递函数的误差分析

Table 3 Error analysis about transfer function

\begin{tabular}{lcc}
\hline & Mean difference $(0 \sim 2 \mathrm{~Hz})$ & Mean difference $(2 \sim 5 \mathrm{~Hz})$ \\
\hline MCAP & 0.0744 & 0.1548 \\
GTF & 0.1630 & 2.4375 \\
NPMA & 0.1144 & 2.2553 \\
\hline
\end{tabular}

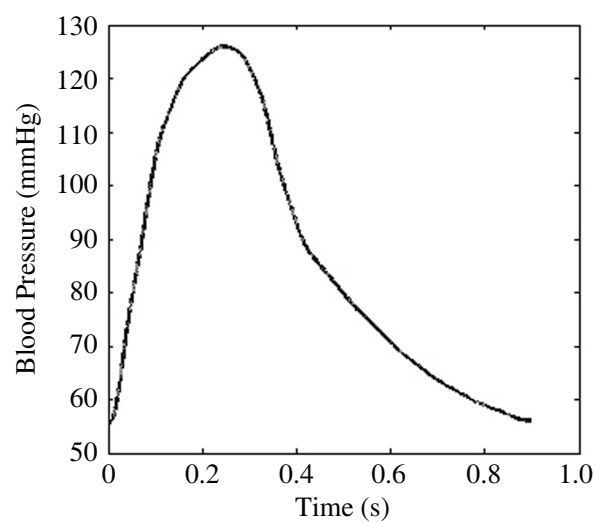

(a)

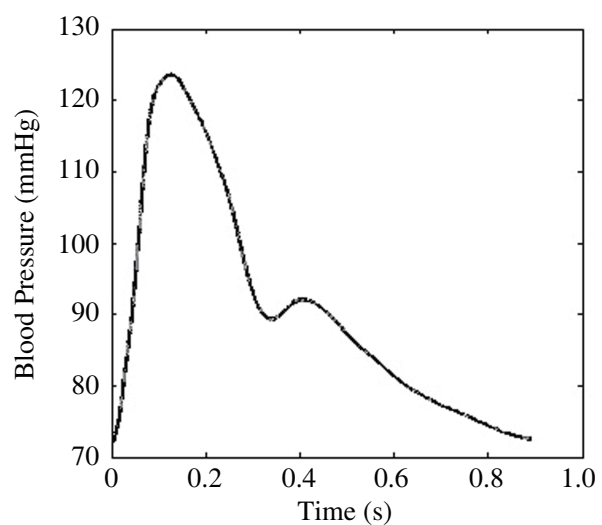

(b)

图 9 桡动脉末端脉搏波波形

Figure 9 The pulse wave of radial artery. (a) The pulse wave of subject whose ID is 10202; (b) The pulse wave of subject whose ID is 317878 
表 4 主要动脉血管的的参数

Table 4 The parameters of blood vessels

\begin{tabular}{ccccccccc}
\hline \multirow{2}{*}{ Vessel } & \multicolumn{3}{c}{ ID:10202 } & & \multicolumn{3}{c}{ ID:317878 } \\
\cline { 2 - 3 } & Compliance & Resistance & Inertia & & Compliance & Resistance & Inertia \\
\hline Ascending aorta & 0.17995 & 0.000105 & 0.000179 & & 1.166300 & 0.000008 & 0.000022 \\
Radial artery & 0.003323 & 3.146500 & 0.075393 & & 0.021537 & 0.227050 & 0.009209 \\
\hline
\end{tabular}

首先, 我们采用桡动脉脉搏波波形对被试心血管个体差异进行定量区分. 近 30 年来, 以脉图面积 变化为基础的脉搏波波形特征量 $\mathrm{K}$ 值被认为是区分个体心血管特性的重要、简单的指标而得到国内 外广泛关注 ${ }^{[23,24]}$. 罗志昌 ${ }^{[25]}$ 等人通过动物实验、临床试验和数学模型计算得到如下结论: 随着患者 年龄增大, 血管顺应性变差, 血液粘度提高, 血管阻力升高, 脉搏波波形中的反射波与主波融合, 主波 变宽大, $\mathrm{K}$ 值升高. $\mathrm{K}$ 值 ${ }^{[25]}$ 的定义为: $K=\frac{P_{\mathrm{m}}-P_{\mathrm{d}}}{P_{\mathrm{s}}-P_{\mathrm{d}}}$. 其中, $P_{\mathrm{m}}$ 为平均血压, $P_{\mathrm{d}}$ 为舒张压, $P_{\mathrm{s}}$ 为收缩压. 因此, 可知 ID 为 10202 的被试的脉搏波波形系数为 $\mathrm{K}=0.4324$, ID 为 317878 的被试的脉搏波的波形 系数为 $\mathrm{K}=0.3661$, 即 ID 为 10202 的被试的外周阻力高于被试 ID 为 317878 的被试, ID 为 10202 的被 试的血管顺应性差于 ID 为 317878 的被试, ID 为 10202 的被试的血液粘度差于 ID 为 317878 的被试.

其次, 我们通过 MCAP 法的心血管模型对被试中心动脉、桡动脉的血管特性 (血管顺应性、血流 阻力和血液流动惯量) 做定量分析, 见表 4 . 由表 4 数值可知: 1) 升主动脉的血管顺应性高于桡动脉血 管顺应性、升主动脉的血流阻力小于于桡动脉的血流阻力、升主动脉的血液流动惯量高于桡动脉的血 液流动惯量. 2) ID 为 317878 的被试的血管特性优于 ID 为 10202 的被试的血管特性: 即 ID 为 317878 的被试的血管顺应性优于 ID 为 10202 的被试; ID 为 317878 的被试的血流阻力低于 ID 为 10202 的 被试;ID 为 317878 的被试的血液流动惯量, 即血液的粘性低于 ID 为 10202 的被试. 上述结论与 $\mathrm{K}$ 值 法定性分析结论相同, 且说明 MCAP 法的心血管模型能以定量形式体现不同血管的血管特性, 具有重 要的临床应用价值. 我们已与北京医院心内科开展心血管模型参数与心血管疾病相关性的研究.

\section{3 误差分析}

观察表 3, 模型法对应传递函数的幅频响应虽优于 GTF 法和 NPMA 法, 但与侵入式法对应传 递函数幅频响应相比仍有一定误差, 我们认为主要由测量误差和系统误差共同引起. 测量误差主要是 指: 受采集条件限制, 被试者的桡动脉和中心动脉的血压波形数据并非同步采集, 而是用侵入式方法 在约 2 分钟内依次采集获得的. 因此采用公式 (27) 估计桡动脉模型参数时, 桡动脉始端压力脉搏波 $P_{22}\left(t, x_{\mathrm{start}}\right)$ 与桡动脉末端流量脉搏波 $Q_{22}\left(t, x_{\mathrm{end}}\right)$ 并不一一对应. 虽然我们认为在短时间内, 脉搏波 波形变化不大, 但这必然在计算模型参数及推导传递函数中产生误差.

系统误差主要是指模型推导过程中, 所做的假设和近似引起的误差 (虽然为了模型的可计算性和 与生物医学参数的一致性, 这些假设和近似是必须的). 例如, MCAP 法估计各血管模型参数 (血管顺 应性、血液流动惯量及血液流动阻力) 时 (公式 (23)) , 假设人体各段动脉血管的长度、半径、壁厚度 及杨氏模量的比值不变. 这将影响心血管模型的精度, 进而影响中心动脉一桡动脉血压传递函数和中 心动脉血压波形的精度.

\section{5 总结}

总结本研究, MCAP 法从理想弹性血管微元的粘性流体力学出发, 完整地提出并推导了一个可计 
算的、与生物医学参数一致的、具有理论基础的人体血管网络模型. 通过分析桡动脉起始端与末端血 压波形, 估计血管和血液参数 (外周阻力、血液流动惯量及血管顺应性), 实现了心血管网络模型参数 的个性化方法. 进而实现无创中心动脉血压估计: 计算中心动脉 一 桡动脉传递函数, 由传递函数和桡 动脉血压计算中心动脉血压. MCAP 法通过对桡动脉脉搏波的分析, 考虑了不同患者的心血管个性化 (血管顺应性、血液流动惯量及血流阻力) 差异. 50 例患者的实验表明, 本方法可以根据被试者的脉搏 波信号调整模型参数, 由模型计算对应的中心动脉 一 桡动脉传递函数幅频响应与侵入式方法对应的 传递函数幅频响应相似. 通过 MCAP 法得到的中心动脉血压波形优于通过 GTF 法和 NPMA 法得到 的结果, 求得的收缩压与舒张压有着很好的准确性与稳定性, 且收缩压和舒张压计算结果符合 AAMI 标准 (误差均值 $\leqslant 5 \mathrm{mmHg}$; 误差标准差 $\leqslant 8 \mathrm{mmHg}$ ). 下一阶段, 我们计划改进模型结构和参数估计 方法, 提高中心动脉血压计算结果的准确性和稳定性, 并研发相应设备, 投入临床试验, 以期我们的研 究成果为人类心血管病的防治有所贡献.

\section{参考文献}

1 Williams B, Lacy P S, Thom S M, et al. Differential impact of blood pressure - lowering drugs on central aortic pressure and clinical outcomes principal results of the conduit artery function evaluation (CAFE) study. Circulation, 2006, 113: 1213-1225

2 Mancia G, de Backer G, Dominiczak A, et al. 2007 guidelines for the management of arterial hypertension: the task force for the management of arterial hypertension of the European society of hypertension (ESH) and of the European society of cardiology (ESC). Eur Heart J, 2007, 28: 1462-1536

3 O'Rourke M F, Seward J B. Central arterial pressure and arterial pressure pulse: new views entering the second century after Korotkov. Mayo Clin Pro, 2006, 81: 1057-1068

4 Williams B, Lacy P S, Yan P, et al. Development and validation of a novel method to derive central aortic systolic pressure from the radial pressure waveform using an N-point moving average method. J Am Coll Cardiol, 2011, 57: 951-961

5 Reymond P. Pressure and Flow Wave Propagation in Patient-Specific Models of the Arterial Tree. Geneva: école polytechnique fédérale de lausanne, 2011, 1-120

6 Formaggia L, Quarteroni A, Veneziani Eds A. Cardiovascular Mathematics: Modeling and Simulation of the Circulatory System. Milano: Springer-Verlag, 2009. 1-522

7 Grinberg L, Cheever E, Anor T, et al. Modeling blood flow circulation in intracranial arterial networks: a comparative 3D/1D simulation study. Ann Biomed Eng, 2011, 39: 297-309

8 Varghese S S, Frankel S H. Numerical modeling of pulsatile turbulent flow in stenotic vessels. J Biomech Eng, 2003, 125: 445-460

9 Reymond P, Merenda F, Perren F, et al. Validation of a one-dimensional model of the systemic arterial tree. Am J Physiol-Heart C, 2009, 297: H208-H222

10 Matthys K S, Alastruey J, Peiró J, et al. Pulse wave propagation in a model human arterial network: Assessment of 1-D numerical simulations against in-vitro measurements. J Biolmech, 2007, 40: 3476-3486

11 Formaggia L, Lamponi D, Tuveri M, et al. Numerical modeling of 1D arterial networks coupled with a lumped parameters description of the heart. Comput Method Biomec, 2006, 9: 273-288

12 Frank O. Die grundform des arteriellen pulses. Z Biol, 1899, 37: 483-526

13 Westerhof N, Lankhaar J W, Westerhof B E. The arterial windkessel. Med Biol Eng Comput, 2009. 47: 131-141

14 Tsanas A, Goulermas J Y, Vartela V, et al. The Windkessel model revisited: a qualitative analysis of the circulatory system. Med Biol Eng Comput, 2009. 31: 581-588

15 Liu Z R. Cardiovascular Fluid Dynamics. Shanghai: Fudan University Press, 1986. 1-357 [柳兆荣. 心血管流体力学. 上 海: 复旦大学出版社, 1986. 1-357] 
16 Feng H B, Ning L. Arterial compliance and its influencing factors. Med Eq, 2007, 20: 31-33 [冯海波, 孙宁玲. 动脉顺应 性及其影响因素. 医疗装备, 2007, 20: 31-33]

17 John D, Anderson, Wu S P, et al. Computational Fluid Dynamics and Its Application. Beijing: China Machine Press, 2007. 1-371 [约翰 D, 安德森, 吴颂平, 等. 计算流体力学基础及其应用. 北京: 机械工业出版社, 2007. 1-371]

18 Bi X F. Human body upper limb vascular system circuit model and simulation. Dissertation for Master Degree. Chongqing: Chongqing University, 2011 [毕喜飞. 人体上肢血管系统电路模型及仿真研究. 硕士学位论文. 重庆: 重庆大学, 2011]

19 Stergiopulos N, Young D F, Rogge T R. Computer simulation of arterial flow with applications to arterial and aortic stenoses. J Biomech, 1992, 25: 1477-1488

20 Wang J J, Parker K H. Wave propagation in a model of the arterial circulation. J Biomech, 2004, 37: 457-470

21 Westerhof N, Bosman F, de Vries C J, et al. Analog studies of the human systemic arterial tree. J Biomech, 1969, 2: $121-143$

22 Chen C H, Nevo E, Fetics B, et al. Estimation of central aortic pressure waveform by mathematical transformation of radial tonometry pressure validation of generalized transfer function. Circulation, 1997, 95: 1827-1836

23 Baker P D, Westenskow D R, Kück K. Theoretical analysis of non-invasive oscillometric maximum amplitude algorithm for estimating mean blood pressure. Med Biol Eng Comput, 1997, 35: 271-278

24 Voelz M. Measurement of the blood-pressure constantk, over a pressure range in the canine radial artery. Med Biol Eng Comput, 1981, 19: 535-537

25 Luo Z C, Zhang S, Yang Y M. Pulse Wave Engineering Analysis and Clinical Application. Beijing: Science Press, 2006. 1-189 [罗志昌, 张松, 杨益民. 脉搏波的工程分析与临床应用. 北京: 科学出版社, 2006. 1-189]

\title{
Modeling human vascular networks and estimation of central aortic pressure
}

\author{
JIANG Sheng ${ }^{1}$, JI LianYing ${ }^{1}$, WANG Fang ${ }^{3}$, SUN YingFei $^{1}$, ZHANG ZhiQiang $^{2}$, JI FuSui $^{3}$, \\ LIU Bing ${ }^{3}$, Zhang WenDuo ${ }^{3}$ \& WU JianKang ${ }^{1 *}$,
}

1 University of Chinese Academy of Sciences, Beijing 100190, China;

2 Imperial College, London SW72AZ, U.K.;

3 Beijing Hospital, Beijing 100730, China

*E-mail: jiankangwu@gmail.com

\begin{abstract}
It becomes a common understanding that central aortic systolic and/or pulse pressures (CAP) provide direct and accurate prediction for cardiovascular structural damage and cardiovascular outcomes. Existing methods for CAP measurement and estimation, namely, method based on general transfer function, and N-point moving averaging method, are rather empirical, and lack of theoretical foundation. Those methods also have inherent drawback that there are not personalized, and missing individual aortic characteristics. Here in this paper, we present a novel method of model-based central aortic pressure measurement and computing, referred to as MCAP. MCAP has solid theoretical foundation; a novel human aortic network model is proposed and developed based on viscous fluid mechanics theory. In MCAP, model parameters of individuals are computed from measured pulse wave of radial artery and brachial artery, the central aortic - radial artery transfer function is derived from the model, and finally, the central aortic pressure and pulse wave are obtained. We conducted an experiment at Cardiovascular Department of Beijing Hospital. Experimental results on 50 test cases have shown better performance of MCAP with comparison to GTF and N-point average method $\left(r_{\mathrm{MCAP}}=0.9667, r_{\mathrm{GTF}}=0.9025\right.$, $\left.r_{\mathrm{NPMA}}=0.8099\right)$. The MCAP accuracy $(\mathrm{Mean}(\mathrm{SBP} / \mathrm{DBP})=-3.9 \mathrm{mmHg} / 3.6 \mathrm{mmHg}, \mathrm{Stdev}(\mathrm{SBP} / \mathrm{DBP})=$ $7.7 \mathrm{mmHg} / 2.9 \mathrm{mmHg}$ ) of central artery systolic pressure and diastolic pressure is up to the standard of AAMI
\end{abstract}


$($ Mean $\leqslant 5 \mathrm{mmHg}, \mathrm{Stdev} \leqslant 8 \mathrm{mmHg})$.

Keywords blood pressure measurement, human artery model, central aortic pressure, viscous fluid mechanics

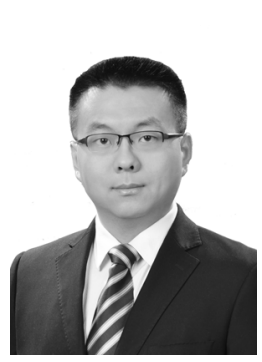

JIANG Sheng received his $\mathrm{Ph} . \mathrm{D}$ degree in Conmunication and Information processing from University of Chinese Academic of Sciences in 2013. His research interest includes mathematical modeling and signal processing.

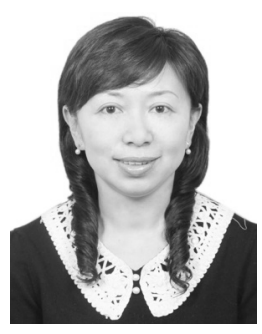

WANG Fang received her Bachelor's degree in Clinical Medicine form Hunan Medical University in 1987, the Master and M.D degrees in Cardiology from the Chinese Academy of Medical Sciences and Peking Union Medical College in 2002 and 2005, respectively. Dr. Wang is a senior researcher at Beijing Hospital, associate director of Department of Cardiology and Clinical Pharmacology Institute, director of Echocardiography. Her research interest includes echocardiography diagnosis and therapy of the cardiovascular diseases. She is committee member of several professional societies, including: Echocardiography Professional Committee, Ultrasound Medical Engineering Society; Echocardiography Working Committee, Chinese Medical Doctor Association, Cardiovascular Physician Branch.

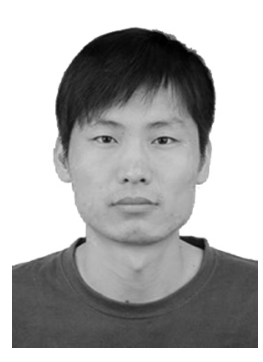

JI LianYing received his Ph.D degree in Signal and Information processing from Beijing Institute of Technology in 2009. Currently $\mathrm{He}$ is a lecturer at University of Chinese Academic of Sciences and a member of IEEE. His research interests include biomedical signal processing.

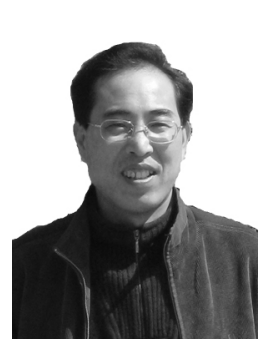

SUN YingFei received his BS degree (1987) and an MS degree (1996) both in mathematics and from Beijing Normal University. He obtained his Ph.D. in Applied Mathematics from Beijing Institute of Technology in 1999. He engaged in a postdoctoral research in the fields of Pattern Recognition and Bioinformatics in Tsinghua University between 2000 and 2002. He is professor at University of Chinese Academy of Sciences. His research area includes Pattern Recognition, Intelligent Systems and Bioinformatics. 